\title{
Frontières
}

\section{Pouvoirs sur la vie et sur la mort}

\section{Les remèdes humanitaires et autochtones dans l'Amazonie brésilienne contemporaine}

\section{Julie Laplante}

Volume 16, numéro 1, automne 2003

Remède ou poison?

URI : https://id.erudit.org/iderudit/1073764ar

DOI : https://doi.org/10.7202/1073764ar

Aller au sommaire du numéro

\section{Éditeur(s)}

Université du Québec à Montréal

ISSN

1180-3479 (imprimé)

1916-0976 (numérique)

Découvrir la revue

Citer cet article

Laplante, J. (2003). Pouvoirs sur la vie et sur la mort : les remèdes humanitaires et autochtones dans l'Amazonie brésilienne contemporaine. Frontières, 16(1),

71-77. https://doi.org/10.7202/1073764ar

\section{Résumé de l'article}

L'article propose une réflexion sur les pouvoirs associés aux remèdes humanitaires et autochtones dans la préservation de la vie comme dans la provocation de la mort. Qu'elle soit attribuée à l'inaccessibilité aux biomédicaments comme le sous-tendent les pratiques de Médecins sans frontières (MSF) ou qu'elle soit allouée, chez les Madija-Kulina, aux pouvoirs que tire le chaman de l'incorporation de certaines plantes, la mort permet aux savoirs biomédicaux cosmopolites et aux savoirs thérapeutiques autochtones de se rencontrer en vue de la défier. Divers témoignages relatifs à l'usage des remèdes ont été recueillis dans le cadre d'un projet de MSF-Hollande auprès des autochtones du Médio Solimões (Amazonie brésilienne) et servent d'appui afin de démontrer les frontières fragiles entre les seuils nocif et bénéfique des remèdes. La rencontre des médecines constitue un moment propice pour lire les risques reliés aux remèdes.
Ce document est protégé par la loi sur le droit d'auteur. L'utilisation des services d’Érudit (y compris la reproduction) est assujettie à sa politique d'utilisation que vous pouvez consulter en ligne.

https://apropos.erudit.org/fr/usagers/politique-dutilisation/ 


\section{Résumé}

L'article propose une réflexion sur les pouvoirs associés aux remèdes humanitaires et autochtones dans la préservation de la vie comme dans la provocation de la mort. Qu'elle soit attribuée à l'inaccessibilité aux biomédicaments comme le sous-tendent les pratiques de Médecins sans frontières (MSF) ou qu'elle soit allouée, chez les Madija-Kulina, aux pouvoirs que tire le chaman de l'incorporation de certaines plantes, la mort permet aux savoirs biomédicaux cosmopolites et aux savoirs thérapeutiques autochtones de se rencontrer en vue de la défier. Divers témoignages relatifs à l'usage des remèdes ont été recueillis dans le cadre d'un projet de MSF-Hollande auprès des autochtones du Médio Solimões (Amazonie brésilienne) et servent d'appui afin de démontrer les frontières fragiles entre les seuils nocif et bénéfique des remèdes. La rencontre des médecines constitue un moment propice pour lire les risques reliés aux remèdes.

Mots clés : remèdes - autochtones humanitaire - Médecins sans frontières Amazonie-Brésil.

\section{Abstract}

This article proposes a discussion of the powers associated to humanitarian and indigenous remedies to preserve life as to provoke death. That the underlying principles of "Médecins sans frontières" (Doctors Without Borders) practices attribute it to a lack of pharmaceuticals or that the indigenous Madija-Kulina attribute it to the powers the shaman gains through the incorporation of certain plants, death permits the encounter of cosmopolitan biomedical knowledge and indigenous therapeutical knowledge in the common goal to defy it. Various testimonies related to the use of remedies have been collected in the context of an MSFHolland project amongst indigenous populations of the Médio Solimões (Brazilian Amazonia) and serve as support to demonstrate the fragile borders between noxious and beneficial effects of remedies. The encounter of forms of curing constitutes a privileged moment to read the risks related to remedies.

Key words: remedies - indigenous populations - humanitarian aid Médecins sans frontières - Amazonia Brazil.

\section{N T E R V E N T I O N}

\section{POUVOIRS SUR LA VIE ET SUR LA MORT Les remèdes humanitaires et autochtones dans l'Amazonie brésilienne contemporaine}

\author{
Julie Laplante, Ph.D., \\ stagiaire postdoctorale en anthropologie, \\ Université de Montréal.
}

Le remède occupe une place prépondérante dans les représentations des causes de la préservation de la vie comme dans celles de la provocation de la mort. Il se fait véhicule par rapport à la vie et à la mort; il apparaît tantôt guérisseur tantôt empoisonneur, selon ses usages ; il sait aussi se faire panacée. Je veux ici mettre en relief deux façons différentes, en lien avec l'usage du remède, de percevoir ces causes reliées à la préservation de la vie comme à la provocation de la mort. Des perceptions humanitaires et autochtones se rencontrent et se côtoient en un même lieu, à savoir à l'intérieur d'un projet de formation de Agente Indìgena de Saùde (AIS, travailleurs en santé autochtones) ${ }^{1}$ mené par l'organisation non gouvernementale (ONG) Médecins sans frontières (MSF) de la section hollandaise (MSF-H) dans des villages MadijaKulina (M-K) du Bas Jurua situé dans le district du Médio Solimões de l'État brésilien de l'Amazonas en Amazonie. L'une de ces façons de voir est véhiculée par des intervenants biomédicaux ${ }^{2}$ et l'autre par des tradipraticiens ${ }^{3}$ des villages en cause.
Le remède constitue un outil commun utilisé par les deux différents groupes d'intervenants de la santé concernés dans leur quête de préserver la vie lorsque des maladies ou des accidents la menacent. Les pouvoirs reliés aux biotechnologies utilisées par les intervenants biomédicaux et les pouvoirs reliés aux plantes savantes ${ }^{4}$ utilisées par les tradipraticiens se présentent de manières très différentes. Pour les intervenants biomédicaux, dans leur intervention humanitaire, la mort est reliée, entre autres, à l'inaccessibilité aux médicaments pharmaceutiques appropriés et adéquats : aucun marché n'en assure la distribution. Pour les autochtones M-K, la mort est attribuée, entre autres, aux pouvoirs incorporés par le chaman à travers les plantes savantes. Lorsque les deux formes de savoirs attenantes à ces perceptions différentes de la mort en lien avec le remède se rencontrent, il y a déséquilibre et fragilisation des modus operandi. Les infections reliées aux morsures de serpent, à la malaria et aux vers intestinaux serviront d'autant d'exemples afin de démontrer les frontières fragiles entre le remède qui guérit et le remède qui empoisonne alors que se rencontrent deux interventions thérapeutiques basées sur des cosmologies très différentes. 


\section{POINT SUR LA MÉTHODE}

Les savoirs humanitaires et autochtones concernés par cet article ont été recueillis dans le cadre d'une ethnographie multisite plus large des projets MSF-H au Brésil où j'ai œuvré comme chercheure durant les années $1998-2000^{5}$, soit aux divers points d'interaction entre les tradipraticiens et les intervenants biomédicaux tant au centre opérationnel de MSF-H à Amsterdam que sur les lieux des bureaux nationaux de MSF-H, au Brésil à Manaus, et sur les lieux de ses projets locaux, à Tefé et à Atalaia do Norte, auprès des autochtones du Médio Solimões et de la vallée de la Javari en Amazonie brésilienne. Les discussions contenues dans cet article se situent à l'intérieur d'un mouvement dans divers sites qui a permis d'observer la construction de savoirs thérapeutiques qui s'inscrivent tout au long d'une trajectoire allant de l'intervention humanitaire aux actes locaux de guérison. Le remède a été considéré comme point de repère et stratégie théorique, pratique et technique. Il a été à la base de la formulation des questions d'entretiens, de ce qui a attiré mon attention lors de l'observation participante et lors de la collecte et de la lecture des documents pertinents. Il a constitué un objet dont je pouvais suivre le trajet et autour duquel se construisaient des savoirs dans les divers sites visités. Le remède, qui est ici aussi bien médicament pharmaceutique, icône par excellence de la biomédecine adoptée par les ONG humanitaires, que plante médicinale, icône de la richesse des médecines autochtones traditionnelles, a servi à cerner un ensemble de savoirs particuliers dans chaque contexte et a servi d'outil pour saisir la dynamique de la rencontre des savoirs dont je propose ici une lecture particulière.

\section{POUVOIR DE VIE ET DE MORT SANS FRONTIĖRES}

Dans l'utopie humanitaire, l'accès aux médicaments pharmaceutiques est gage de survie alors que l'inaccessibilité de ceux-ci signifie mort probable. Les médicaments pharmaceutiques constituent l'outil essentiel des interventions en santé internationale de MSF. De fait, "les médicaments essentiels constituent la fondation pour presque tous les programmes de santé publique » (Pécoul et al., 1999, p. 361), soit le moyen principal utilisé pour atteindre les objectifs de l'Organisation mondiale de la santé (OMS) de procurer la santé pour tous.

C'est à l'intérieur de cette envolée qu'une Campagne d'accès aux médicaments essentiels aura été lancée par MSF-USA dans les années 1998-1999: "MSF is campaigning for change to ensure that disadvantaged populations get the medicines they need ${ }^{6}$. »
Cette campagne est issue de trois constats la fraude dans la qualité des médicaments livrés par plusieurs pays, les biais commerciaux dans l'industrie pharmaceutique et, principalement, l'injustice sociale quant à l'accès aux médicaments. C'est au nom des droits de l'homme que la campagne poursuit alors des objectifs d'équité, dont celui d'humaniser les savoirs et les pratiques de l'industrie pharmaceutique et du système de santé à l'échelle du globe en exerçant des pressions aux niveaux politique, économique et sur le plan de la recherche et du développement. La cause est incontestable : humaniser les intérêts commerciaux de l'industrie pharmaceutique qui guide les recherches, fixe les prix des produits et établit leur réseau de distribution.

Mais l'objectif d'aider les populations désavantagées à se procurer les médicaments pharmaceutiques dont elles ont besoin (ou dont on crée le besoin) est plus controversé et soutient, malgré tout, l'industrie pharmaceutique. L'acte biomédical humanitaire «neutre, a-politique, a-religieux » est plus ambigu qu'il ne le laisse paraître. Selon cette perspective humanitaire, la mort physiologique dépend d'injustices commerciales/ sociales ou d'un unprofitable market syndrome $^{7}$, soit de l'impossibilité d'avoir accès, au moment opportun, aux médicaments ou aux biotechnologies appropriés. Les médicaments pharmaceutiques deviennent la solution privilégiée aux problèmes de santé, négligeant à la fois les limites des bienfaits de ces substances, l'existence des effets secondaires des médicaments et ignorant la vie hors de la technique, le contexte étranger au savoir biomédical.

Cette perspective sous-tend les pratiques des intervenants biomédicaux de MSF sur les lieux des projets brésiliens même si la réalité nationale apporte des nuances quant au rôle de panacée du médicament pharmaceutique au moment où les enjeux de la biopiraterie et de la propriété intellectuelle ${ }^{8}$ soulèvent la question des savoirs et des remèdes autochtones. Bien qu'officiellement endossée par l'OMS depuis 1978, la reconnaissance des savoirs et remèdes autochtones demeure minimale et timide dans les interventions humanitaires. C'est l'usage du médicament pharmaceutique qui prime, d'abord parce qu'il relève de l'expertise des intervenants biomédicaux, mais aussi parce qu'il est largement revendiqué par les populations autochtones conquises par l'exotisme et l'effet panacée du produit. Le médicament pharmaceutique est ce qui constituera, pour les intervenants MSF, «le pouvoir de dire et d'agir » (Foucault, dans Dreyfus et Rabinow, 1984, p. 309) sur la vie et sur la mort dans les villages autochtones. Ces intervenants biomédicaux, et ceux qu'ils forment, dépendront presque

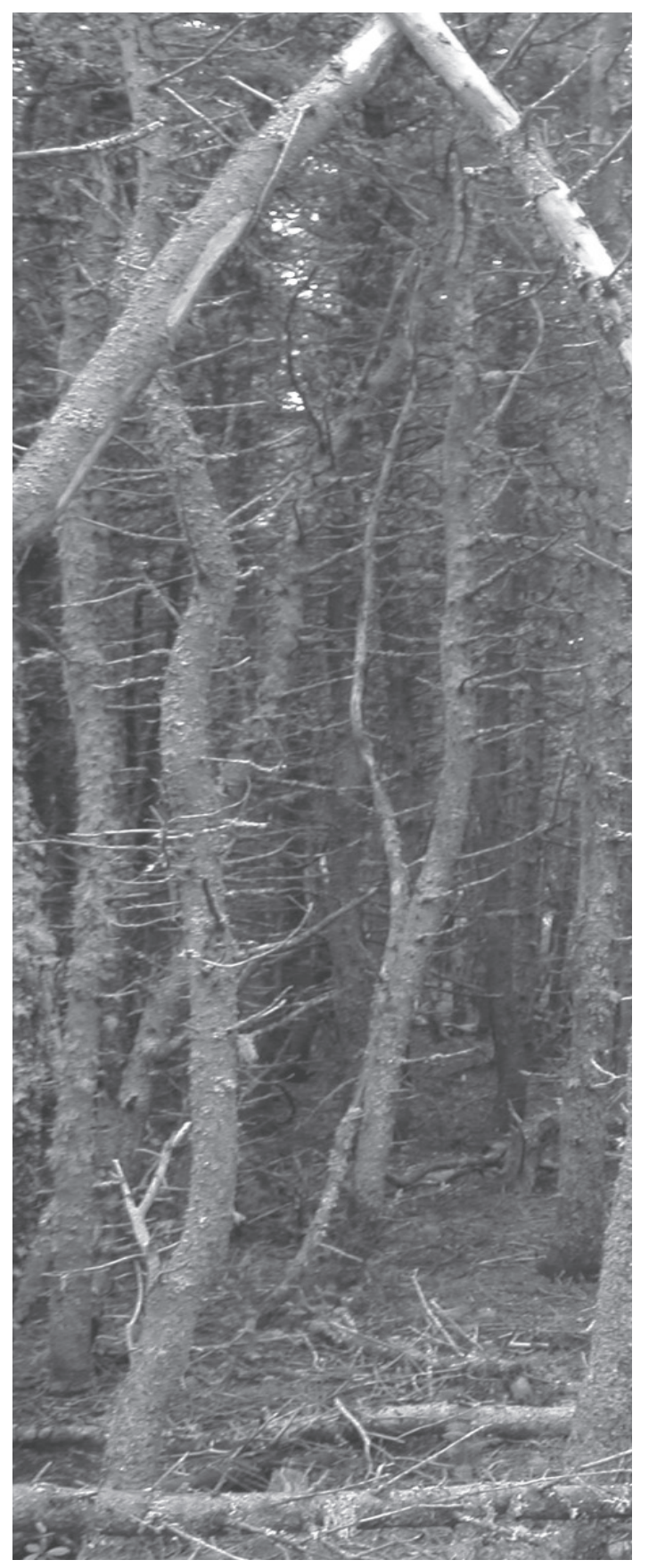

exclusivement des biotechnologies dans l'exercice de leurs pratiques. Le biomédicament constitue l'outil essentiel des programmes de formation du personnel local en biomédecine. Le AIS formé, entre autres, par des infirmières MSF, dépend entièrement du médicament pharmaceutique réinterprété dans un modèle de "commodification » de la santé ${ }^{9}$. Sans médicaments, il n'est plus intervenant en santé. Cette réalité conduira certains AIS M-K à dire que la maladie c'est "quand il n'y a pas de médicament», quand ils ne peuvent plus intervenir. D'autres établiront un lien d'équivalence entre prendre un médicament et être en bonne santé. Plusieurs AIS affirment qu'avant d'avoir suivi les cours de formation biomédicale (qui peuvent durer une 


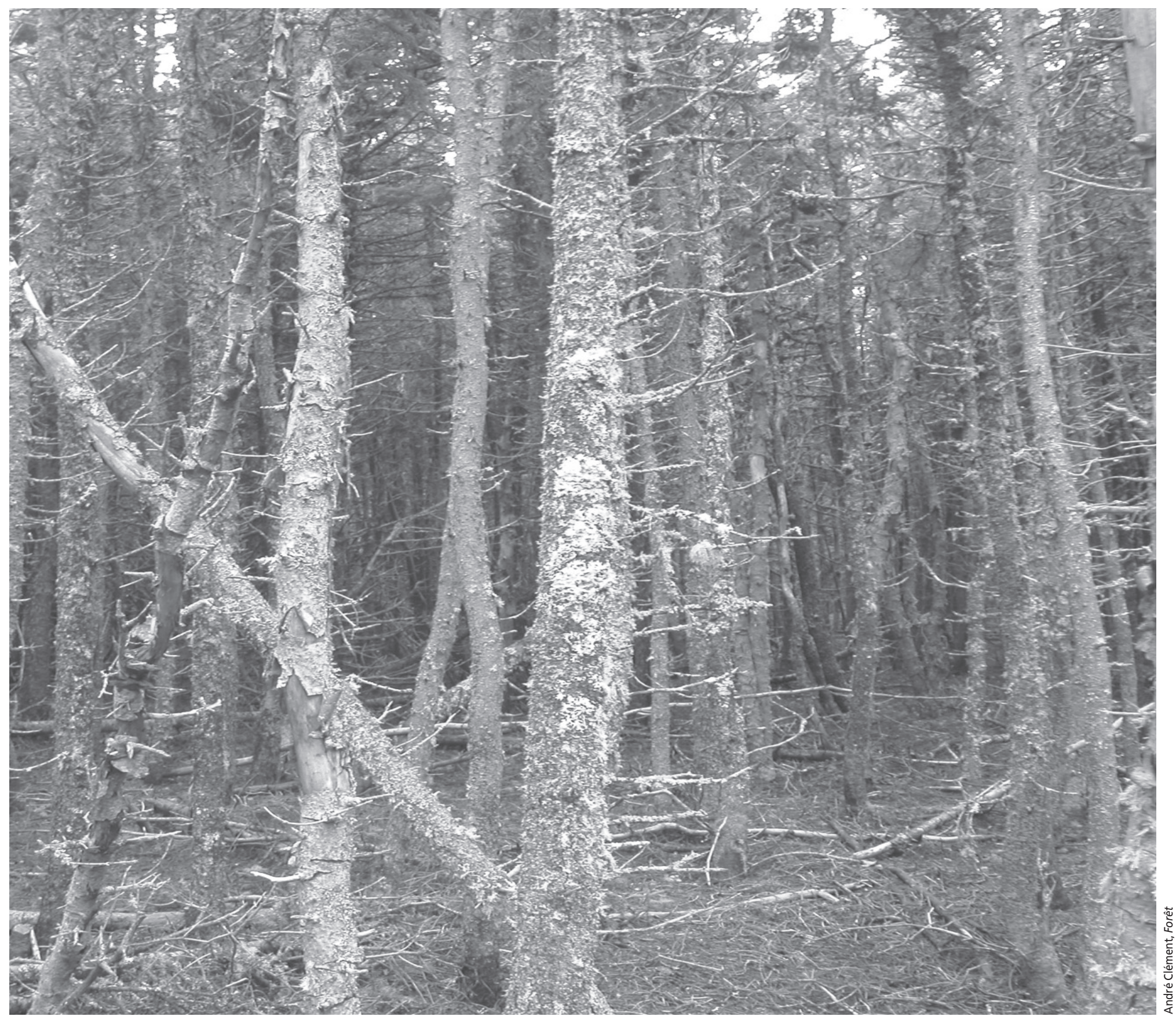

dizaine de jours par année), ils ne savaient rien; mais depuis qu'ils sont plus instruits (parfois après une ou deux sessions de cours), la santé des villageois s'est améliorée! Cette affirmation dénote une forme de croyance en la magie des médicaments pharmaceutiques.

Les intervenants biomédicaux (humanitaires et AIS) deviennent facilement interchangeables et leurs liens aux technologies priment sur leur compétence. Leur rôle se réduit aux fonctions entourant l'utilisation des biotechnologies, l'acquisition de leur expérience étant aliénée ou médiatisée par un objet. Dans la pratique journalière des intervenants biomédicaux dans les villages autochtones, l'intervention biomédicale, privée de ses infrastructures, repose pour ainsi dire uniquement sur le médicament et c'est à ce dernier que revient le rôle de sauver la vie ou de laisser la mort faire son œuvre, l'inaccessibilité aux médicaments pharmaceutiques signifiant un taux accru de mortalité.

\section{POUVOIR DE VIE ET DE MORT MADIJA-KULINA (M-K)}

Les perceptions des causes de la préservation de la vie et de la provocation de la mort en lien avec les remèdes se présentent de manières très différentes au cœur des villages autochtones $\mathrm{M}-\mathrm{K}$ dont la cosmologie est tout autre que celle liée à l'utopie humanitaire. En 1998, les populations autochtones M-K du Bas Jurua comptent plus de 300 personnes réparties entre quatre villages du territoire du lac Ualá. Elles se seraient établies sur ce territoire au début des années 1980 après de nombreux déplacements et contacts sporadiques avec divers acteurs de la société cosmopolite ${ }^{10}$. Ces villages se situent à plus de deux jours de rame (en canot) de la ville la plus proche offrant des services hospitaliers et dépendent des bateaux des ministères de la Santé et de la Justice pour s'y rendre plus rapidement (ce qui signifie un minimum de quelques heures). Ces populations sont donc en relative isolation par rapport aux soins de santé biomédicaux reliés à la société nationale et internationale.

Dans les villages $\mathrm{M}-\mathrm{K}$ du lac Ualá, des savoirs liés à la biomédecine ont d'abord été introduits par des acteurs de la société 
cosmopolite (commerçants, ouvriers de Petrobràs) qui n'ont pas de véritable expertise biomédicale mais qui en connaissent des concepts de base et, surtout, en utilisent les outils. Depuis 1992, un représentant de la Fundaçao Nacional do Indio (FUNAI, Fondation nationale de l'Indien) du ministère brésilien de la Justice habite plusieurs mois par année un poste de santé construit dans le village Cumarú. Cette personne n'a aucune formation biomédicale, mais gère une pharmacie de biomédicaments et véhicule, par le fait même, des principes biomédicaux de base. Depuis quelques années, il y a aussi des autochtones qui assument le rôle de AIS, dont un AIS d'origine brésilienne, marié à une femme M-K, et qui, connaissant lui aussi quelques concepts biomédicaux de base, prend la relève du représentant de la FUNAI lorsque celui-ci s'absente du poste de santé. C'est depuis février 2000 qu'une équipe du Distrito Sanitario Especial Indìgena (DSEI, District sanitaire spécial autochtone) du ministère brésilien de la Santé a pris la relève définitive de la gestion du poste de santé de la FUNAI. L'équipe compte une infirmière novice et deux auxiliaires infirmières qui travaillent sous sa tutelle.

Les savoirs thérapeutiques autochtones s'ancrent d'abord dans la tradition locale et l'expérience de la population en général, des chamans, des hommes-tabac et des sages-femmes, mais s'articulent aussi à une lutte politique pro-autochtone (indigénistes) ou de sensibilité culturelle (anthropologues). Depuis 1991, trois indigénistes de l'ONG brésilienne Conselho Indigenista Missionário (CIMI, Conseil missionnaire autochtone) habitent huit mois par année les villages Pau Pixuna et Cumarú. Elles agissent en tant que gardiennes de la culture ayant, entre autres, les objectifs de revaloriser l'utilisation des remèdes locaux afin de diminuer la dépendance aux remèdes allopathiques et de stimuler l'intégration du chaman. Des anthropologues de passage jouent aussi un rôle dans la mise en valeur des savoirs thérapeutiques locaux ${ }^{11}$. J'ai moi-même, accompagnée de membres de l'União dos Nações Indìgenas de Tefé (UNITefé, Union des nations autochtones de Tefé), participé à rehausser l'importance des savoirs thérapeutiques locaux avant que soit initié le projet de MSF-H. Un rôle d'herboriste émerge du métissage de ces pratiques locales et luttes cosmopolites, en plus de la mise sur pied de cours de Médecine traditionnelle (UNI-Tefé, CIMI) et de AIS, conduisant au glissement des plantes médicinales aux côtés des médicaments pharmaceutiques à l'intérieur d'un modèle de commodification de la santé.

Dans ce contexte traditionnel enchevêtré de diverses influences cosmopolites, les perceptions des causes de la préservation de la vie et de la provocation de la mort sont évoquées à l'intérieur de trois catégories de maladie / traitement: dori / plantes savantes, maladies naturelles/jemedsi (remèdes de la terre) et doença mesma (maladie en soi) ou doença do Branco (maladie des Blancs) / médicaments pharmaceutiques. Les étiologies de ces maladies / traitements relèvent respectivement $\mathrm{du}$ social, des éléments de la nature et du contact avec la société cosmopolite, mais, en dernière instance, la préservation de la vie et la provocation de la mort ne sont attribuées ni à l'une ni à l'autre cause de maladies ou type de remèdes, mais à des forces de l'au-delà. Un regard plus pointu du couple dori/plantes savantes permettra de lire les représentations locales de la vie et de la mort en lien avec les remèdes alors qu'un regard sur le couple maladies naturelles / jemedsi aidera à comprendre comment ces représentations s'articulent (ou non) aux maladies en soi / médicaments pharmaceutiques.

Le pouvoir du chaman sur la vie et la mort est lié à l'incorporation et à la transmission du dori. Le dori se manifeste comme un objet dur, quelque chose sous la peau qui trouble la circulation du sang. Il apparaît le plus souvent à la suite d'actes sociaux qui ont choqué, qui ont rendu jaloux ou qui ont créé une injustice sociale irréparable. Il est transmis par les chamans, parfois par les chamans du même groupe tribal que la victime, mais le plus souvent par des chamans de groupes voisins concurrents. Il se transmet souvent entre chamans en guise de règlement de comptes entre les groupes, mais aussi entre divers membres d'un même village, par un chaman acolyte $^{12}$.

Le pouvoir de guérir est transmis lors d'une initiation à l'occasion de laquelle le dori entre dans le corps de l'initié par l'entremise des esprits tokorine et lui donne les pouvoirs d'agir (ou non) en tant que chaman, c'est-à-dire de communiquer avec les esprits, de visualiser, diagnostiquer et de retirer ou donner le dori. Les chants permettent aux chamans d'incorporer les esprits; ils sont la manifestation même de ces esprits rendus présents au groupe à travers le corps du chaman ${ }^{13}$. Révéler la teneur des chants (considérés le langage des esprits qui passent à travers le corps du chaman) comme révéler les pratiques du chaman pourrait entraver l'intervention des esprits. Le succès de la cure du chaman dépend aussi de l'incorporation de deux plantes savantes, à savoir le rami ${ }^{14}$ et le tabac. Leur incorporation permet l'acquisition des savoirs des esprits puissants dépositaires et gardiens jaloux du savoir de ce monde. Ces deux plantes qui enseignent donnent au chaman les capacités de voir, de donner et de retirer le dori. C'est donc premièrement dans le corps même que s'acquièrent les pouvoirs de guérison du chaman (par opposition aux pouvoirs de l'intervenant biomédical qui s'acquièrent d'abord par une formation académique, à laquelle s'ajoute ensuite l'expérience); c'est aussi à travers le corps que la maladie de l'autre est ressentie et guérie et par le corps que la maladie est transmise à l'autre.

Le rami et le tabac constituent deux sources de savoir/pouvoir des tradipraticiens lors du processus de guérison. Le rami permet d'identifier le mal grâce à des visions, le tabac, d'extraire le mal au moyen des fumigations. Le chaman lui-même et l'homme-tabac «soufflent pour voir». Ces deux plantes se consomment toujours à l'intérieur d'un rituel, évitant ainsi les risques mortels de leur surconsommation. Ces risques font souvent partie de l'initiation des chamans qui doivent frôler la mort afin d'obtenir leurs pouvoirs. S'intoxiquer partiellement avec l'une et/ou l'autre de ces plantes savantes permet d'approcher le monde des esprits et d'ainsi renforcer la personne. Lorsque des proches veulent participer à la cure et consommer le rami (excluant la plupart du temps le malade), un chaman responsable vérifie leur pouls afin de s'assurer qu'il n'y ait pas d'intoxication au produit (un pouls irrégulier ou trop rapide indiquerait un excès), mais de manière générale seul le chaman ingère les plantes et tout se fait par son entremise (et celle de l'homme-tabac). L'incorporation des plantes savantes par le chaman constitue un moyen pouvant indiquer le chemin vers le maintien de la vie aussi bien que vers la maladie et la mort. Si la personne qu'il traite meurt, il peut en être tenu responsable et être menacé de mort et / ou tué par d'autres chamans. C'est en voyant la maladie (à partir de sensations corporelles faisant suite à la consommation du rami) que le chaman (et l'homme-tabac) découvre s'il peut retirer ou non cette maladie; s'il ne peut pas la retirer, et que la personne ne meurt pas, elle peut alors être définie comme une maladie naturelle ou une maladie en soi nécessitant d'autres formes d'interventions.

Les maladies naturelles peuvent avoir les mêmes symptômes que le dori ou la maladie en soi, mais leurs causes ne sont pas attribuées à un acte social ou au contact avec la société cosmopolite mais à l'environnement local : à l'air, aux animaux terrestres et aquatiques, au temps ou aux insectes. Le traitement des maladies naturelles relève normalement du domaine profane des jemedsi. La langue orale des M-K, le kulina, n'a que ce vocable pour signifier les substances à base de plantes, d'animaux 
ou de minéraux utilisées pour traiter les maux concrets du quotidien, s'y ajoutent aujourd'hui divers produits cosmopolites, dont les médicaments pharmaceutiques.

En général, ce sont les femmes du village (souvent des sages-femmes plus âgées) qui préparent les jemedsi mais tous les villageois participent et tentent de trouver les remèdes qui fonctionneront. Dans le cas d'un homme d'une trentaine d'années s'étant fait piquer par une raie, par exemple, tous se sont affairés à la recherche des jemedsi qui pouvaient alléger sa douleur. Deux hommes sont partis en canot à la recherche d'un nid de tukandeira (fourmis noires); le nid fourmillant de ces insectes encore vivants et qui piquent sera brûlé et sa fumée devra soulager la douleur. D'autres sont allés chercher de la canne à sucre afin d'en extraire le jus que l'homme boira afin d'alléger la douleur de sa piqûre. Un homme est venu à ma hutte chercher du lait en poudre dans le même objectif. Un autre proposait de mettre du chlorure de sodium dans les breuvages. Avant tous ces recours, plusieurs femmes avaient installé l'homme blessé près du feu et avaient serré trois tourniquets afin d'empêcher le venin de la raie de monter dans le haut de son corps.

Plusieurs principes propres au traitement des maladies naturelles sont conciliables avec les savoirs thérapeutiques populaires reliés au contexte biomédical ou encore sont conciliables avec une construction explicative «rationnelle» de la maladie (non associée au surnaturel) relevant d'un empirisme local. Les intervenants biomédicaux sont beaucoup moins aliénés par ces formes de traitements que par ceux du dori. Les maladies naturelles fournissent un terrain propice à la construction de nouveaux savoirs et c'est surtout autour d'elles que les pratiques thérapeutiques des M-K s'ancrent de plus en plus dans des traitements mixtes locaux et cosmopolites. Les médicaments pharmaceutiques ou tout produit de l'extérieur, en quelque sorte des jemedsi exotiques, s'infiltrent dans ces cures alors que les maladies naturelles se métissent avec les maladies en soi ou les maladies déclarées nouvelles.

Dans les villages visités, de nouvelles recettes combinant des produits récemment introduits (produits alimentaires, produits pharmaceutiques, produits pour les moteurs) et des plantes médicinales communes apparaissent et font aujourd'hui partie des préparations visant la guérison des maladies naturelles. La gazoline, par exemple, connaît plusieurs fonctions thérapeutiques chez les M-K ; elle s'utilise sur les dents douloureuses (on met la gazoline sur du coton que l'on applique sur la dent quelques minutes avant de cracher) avec (ou sans) la fleur de jambù. La gazoline s'utilise aussi contre les piqûres d'insectes, sur les plaies de rougeole, contre les dermatites (directement appliquée sur les plaies pour les assécher). La canne à sucre, récemment introduite dans les villages, sert dans les cas d'empoisonnement et de piqûres de raie (on en boit le jus). Selon les circonstances, le poison (gazoline) peut devenir le remède, et le remède (canne à sucre) devenir l'antidote au poison, ou encore le médicament fortifiant (vitamine C) deviendra poison et provoquera un va-et-vient entre savoirs / pouvoirs traditionnels et savoirs / pouvoirs biomédicaux.

C'est ainsi qu'une femme M-K, ayant consommé le contenu d'une vieille boîte de vitamine $C$ qui descendait la rivière alors qu'elle faisait son lavage, souffre de maux de tête et de nausées graves. La femme consultera les infirmières du DSEI sans succès et ce sera grâce à un rituel de rami mené par un homme-tabac qu'elle se rétablira. Ce sera aussi à l'homme-tabac que reviendra la tâche de trouver remède à certaines maladies liées à la consommation de produits cosmopolites (dont la cachaça, boisson brésilienne alcoolisée à $40 \%$, par exemple, ou encore des produits pharmaceutiques ayant causé préjudice). Les morts infantiles à la suite de campagnes de vaccination ne seront pas attribuées aux vaccins ; ce n'est pas aux substances elles-mêmes que revient le rôle de déjouer ou de donner la mort. Il en reviendra plutôt aux tradipraticiens d'en résoudre la cause avec les esprits, malgré le fait que la vaccination soit dénoncée comme entravant les pouvoirs des chamans et les obligeant à se réinitier afin de retrouver leurs pouvoirs. C'est dans une lutte entre les esprits, ici ceux du dori et ceux du monde cosmopolite incorporés dans la substance injectable, que la mort sera mise à l'épreuve.

\section{FRONTIÈRES FRAGILES ENTRE LES SEUILS NOCIF ET BÉNÉFIQUE DES REMÈDES}

Pour un remède donné, les frontières entre sa capacité de guérir et celle d'empoisonner se fragilisent au moment où celuici se distancie des savoirs qui le soutiennent, au moment où s'écroulent ou se transforment les infrastructures qui supportent son utilisation. Lorsque se croisent biomédecine et médecine traditionnelle, le remède guérisseur risque de devenir un poison. L'usage du médicament pharmaceutique dans des villages autochtones de l'Amazonie brésilienne fait ressortir le paradoxe de l'utilisation de celui-ci hors contexte. Rien ne garantit que, par le biais des interventions humanitaires, la quête du remède guérisseur ne se transforme pas en l'acquisition d'un remède empoisonneur. La longue chaîne d'efficacité qui doit être maintenue afin que le médicament demeure bénéfique dépend de plusieurs facteurs : «recherche et développement d'un agent pharmaceutique approprié, production, contrôle de qualité, distribution, contrôle de l'inventaire, information fiable pour les professionnels de la santé et le public en général, diagnostic, prescription, accessibilité financière, dosage de la drogue, observance et pharmacovigilance » (Pécoul et al., 1999, p. 361) ; une chaîne d'efficacité difficile (pour ne pas dire impossible) à maintenir dans le contexte amazonien contemporain alors qu'elle dépend entièrement de savoirs, de techniques et de produits provenant d'une industrie étrangère. De plus, le médicament nouvellement introduit est réinterprété, ramène à la tradition locale et entraîne de nouveaux risques tant pour les pratiques thérapeutiques locales que pour la santé des individus eux-mêmes. Plusieurs exemples peuvent illustrer la fragilité du seuil bénéfique des remèdes alors que les infrastructures sociales qui les soutiennent sont mises au défi.

Un cas de morsure de serpent illustre la complexité de l'intégration des soins hospitaliers biomédicaux (dont on a vu une meilleure performance dans des hôpitaux situés dans de plus grandes métropoles) dans un contexte local traditionnel et les nouveaux périls que la dépendance à ces soins et services externes peut créer. Un homme M-K s'est fait mordre par un serpent au cours d'une expédition en forêt. Comme la tradition le veut, sa femme, qui l'accompagnait, l'a immédiatement quitté afin de chasser le serpent, qu'elle a tué sur place et ramené en trophée. À l'arrivée au village, les tradipraticiens de l'endroit ont identifié le serpent et préparé un thé de piâo roxo. Traditionnellement, lors d'une morsure de serpent, maladie dite naturelle et très commune, la population du village prend la situation en charge et s'entend pour immobiliser la victime; le traitement se poursuit sur place avec une série de rituels et de préparations à base de plantes. Cette fois, les intervenantes biomédicales du DSEI en poste dans le village ont proposé un traitement en milieu hospitalier lequel, à ce moment, fonctionnait très mal. Le transfert à l'hôpital a interrompu le traitement local, a obligé la victime à se déplacer et ainsi a gaspillé un temps précieux qui a retardé le traitement. Le transport à l'hôpital le plus près a duré plus de trois heures et il s'est avéré que les produits pharmaceutiques nécessaires n'étaient pas disponibles, obligeant la victime à se déplacer jusqu'à l'hôpital de Tefé, soit à un autre six heures de bateau, et où les produits disponibles se sont révélés peu efficaces. Le corps du blessé sera demeuré enflammé pendant trois semaines, alors que, sous le traitement 
traditionnel, l'enflure se résorbe normalement en une semaine. La marginalité des interventions biomédicales non modulées, dans ce cas, aux besoins concrets a produit des effets contraires au bien-être du blessé.

Le cas de la malaria illustre aussi le passage périlleux vers des pratiques biomédicales, les effets de l'abandon des savoirs thérapeutiques locaux ainsi que le seuil nocif du médicament pharmaceutique catalysé lorsque hors contexte. Chez les M-K, la malaria est le prototype des maladies en soi et révèle le glissement d'une maladie de catégorie dite naturelle que l'on peut traiter dans le cadre thérapeutique traditionnel local vers la catégorie maladie en soi, en raison d'une réinterprétation de la maladie en cause (et/ou de sa transformation) comme venant de l'extérieur, donc pour laquelle on fera appel aux soins de santé apportés de l'extérieur. Les visites au laboratoire et les transferts aux hôpitaux sont fréquents et presque automatiques lors du traitement de la malaria. Plusieurs tentatives d'identifier, dans les villages, le type de malaria impliqué afin de sélectionner le médicament pharmaceutique associé échouent en raison du bris du microscope et / ou d'un manque de disponibilité d'un intervenant qui sache lire les lamelles. Les essais d'utilisation des médicaments pharmaceutiques sans la technologie de soutien ont même conduit à des pratiques catalysant la résistance au traitement: en l'absence d'un microscope disponible pour identifier le virus en cause, il arrive fréquemment que deux médicaments de types différents soient consommés simultanément ou successivement afin de s'assurer que toutes les formes de malaria identifiées dans la région (Plasmodium vivax bénigne ou $P$. falciparum fatale) soient ciblées.

C'est à l'insu des principaux utilisateurs que le remède guérisseur de la biomédecine semble atteindre ses limites, ne plus assurer l'effet guérisseur pour lequel il est tant glorifié et se transformer en produit toxique pour l'organisme. Le phénomène grandissant de résistance à tous les médicaments antipaludéens utilisés en Amazonie (chloroquine, quinine, primaquine et méfloquine ${ }^{15}$ ) indique que leur seuil nocif est peut-être atteint. C'est au niveau de la cure que la question se pose quant à l'efficacité des médicaments antipaludéens actuellement sur le marché ; un renouvellement s'imposerait $^{16}$ (Pécoul et al., 1999, p. 364). Si ces médicaments ne guérissent plus, alors ils ne font qu'endommager le foie et rendre plus vulnérable aux crises de malaria. Les relations de pouvoir auxquelles se greffe le savoir biomédical masquent ces limites des médicaments ainsi que la source des substances utilisées, source qui nous ramènerait peut-être à la forêt amazonienne ${ }^{17}$, aux jemedsi autrement utilisés pour guérir la malaria, dont l'écorce du paracarnaùba, le saracura et le melão de São Caetano qui sont les plus fréquemment mentionnés chez les M-K. Les problèmes de résistance aux antibiotiques et aux médicaments contre la malaria apparaissent partout en Amazonie, ainsi qu'une forme de tuberculose résistante aux médicaments après plusieurs années de traitements mal administrés (entre autres, interruption précoce du traitement et surconsommation).

S'il est difficile pour les populations autochtones d'apprécier les médicaments pharmaceutiques à leur juste valeur, et si ces populations ont tendance à considérer ces médicaments dans une catégorie à part, à savoir comme des remèdes exotiques toutpuissants ne possédant que des effets bénéfiques, il en va de même pour certains intervenants biomédicaux qui attribuent aux jemedsi le même rôle de panacée. C'est ainsi qu'une médecin de MSF m'a suggéré de consommer du lait de papaye verte contre les vers intestinaux dont je pensais être infestée. Me méfiant quelque peu, en raison de ma condition plus fragile de femme enceinte, d'effets toxiques éventuels même s'il s'agissait d'un produit naturel, je me suis renseignée auprès de plusieurs tradipraticiens et des femmes du village pour apprendre que, oui, effectivement, le lait de papaye verte pourrait me débarrasser des vers, mais aussi du fœtus. Le remède contre les vers s'avérait un poison mortel pour l'embryon. L'exotisme du produit, et pour les populations autochtones et pour les intervenants humanitaires, aveugle souvent sur la teneur réelle de la substance utilisée, incite de nouveaux usages expérimentaux et le remède qui guérit court un plus grand risque de devenir le remède qui empoisonne.

Bien que tous les tradipraticiens tiennent compte de la toxicité possible des jemedsi, que les chamans M-K s'assurent d'éviter une surconsommation toxique et dangereuse du breuvage rami et / ou du tabac, de même que tous les intervenants biomédicaux savent que la dose assure au biomédicament son effet guérisseur ou son effet poison, l'exotisme d'une nouvelle substance masque souvent, lors de la première rencontre avec elle, ses dangers réels, ses risques d'empoisonnement. Les seuils nocifs et bénéfiques du remède doivent alors être mis en relief. Les remèdes, quels qu'ils soient, doivent éventuellement être démystifiés, contrairement à diverses stratégies de savoir et de pouvoir, contrairement, entre autres, aux stratégies de la médecine humanitaire dans le cas du médicament pharmaceutique lorsqu'il est introduit sans subtilité dans des villages autochtones.

On a vu la Campagne internationale d'accès aux médicaments essentiels de
MSF brandir le médicament comme une garantie presque certaine de survie et attribuer à l'inaccessibilité aux médicaments, à la faillite du marché des médicaments la mort probable. Dans le cadre d'une médecine humanitaire sans frontières, les contextes d'intervention sont si variables que l'on peut s'en remettre à la pénurie de médicaments pour expliquer la mort. Cette conception est rendue possible du fait que les pratiques entourant le médicament pharmaceutique participent à l'utopie humanitaire. Passant sous silence ses effets secondaires parfois fatals ${ }^{18}$, la médecine humanitaire peut ainsi introduire ses remèdes guérisseurs comme des panacées, dissimulant temporairement leurs frontières communes avec les produits toxiques, rompant même le lien avec la tradition biomédicale qui ramènerait à l'ancien terme grec pharmakon, d'où dérive celui de "pharmaceutique », qui signifiait aussi poison (Whyte et al., 2002, p. 6).

Les pouvoirs liés à la médecine humanitaire renvoient à leurs effets thérapeutiques à court terme, mais surtout à leurs liens avec la stratégie de développement inspirée par les variables clés que sont le capital, la technologie et certaines institutions (universitaires, gouvernementales, religieuses...) (Escobar, 1984-1985, p. 386). Ce sont ces relations de savoir / pouvoir qui permettent de comprendre la dynamique de domination des pratiques entourant les médicaments pharmaceutiques par rapport à celles entourant les jemedsi sur la trajectoire humanitaire. Malgré leur exotisme initial, lorsque les pratiques entourant les jemedsi quittent les villages autochtones et circulent sur la trajectoire humanitaire, sans leur infrastructure de soutien liée à l'expérience et à la cosmologie locales, elles perdent leur crédibilité face aux pratiques entourant les médicaments pharmaceutiques, à moins d'être retraduites dans les taxonomies scientifiques ou encore transformées en produits de l'industrie pharmaceutique. Le pouvoir de vie et de mort $\mathrm{du}$ remède est une question économique, politique, sociale et culturelle. Lintervention technobiomédicale sans frontières provient d'une biopolitique particulière pouvant agir à l'échelle du globe sur ce qu'Agamben (1997) appellera la vie nue, soit le simple fait de vivre, commun à tous les êtres vivants, et entraîne avec elle à la fois ses bienfaits et ses périls.

\section{Bibliographie}

AGAMBEN, G. (1997). HOMO SACER. Pouvoir souverain et la vie nue. Paris, Seuil.

BURROUGS, W. (1989). The Job (par D. Odier), Penguin Books. 
CHOMSKY, N. (1994). "Le pillage des ressources intellectuelles », Écologie politique, $\mathrm{n}^{\circ} 9$ (printemps).

DESCOLA, P. (1993). Les lances du crépuscule, Paris, Plon, coll. «Terre humaine».

DESCOLA, P. (1996). "Constructing natures: symbolic ecology and social practice», dans P. DESCOLA et G. PÀLSON (dir.), Nature and Society. Anthropological Perspectives. Londres et New York, Routledge, p. 82-102.

DOBKIN DE RIOS, M. (1972). Visionnary vine: Psychedelic healing in the Peruvian Amazon. San Francisco, Chandler Publishing Company.

DREYFUS H. et P. RABINOW (1984). Michel Foucault. Un parcours philosophique. Paris, Gallimard.

ESCOBAR, A. (1997). "Anthropologie et développement.", Revue internationale des sciences sociales, $\mathrm{n}^{\circ} 154$ (décembre), p. 539560 .

FAULHABER, P. (1994). Relatório de Identificação e delimitação de A.I. Cumarú (Kulina/Madija). Grupo técnico CNPq/ Museu Goeldi, Belém, Parà.

LAPLANTE, J. (2002). Savoirs, pouvoirs et médecines. De l'humanitaire aux autochtones de l'Amazonie brésilienne. Thèse doctorale, Université Laval.

LAPLANTE, J. (sous presse). «Le médicament aux frontières des savoirs humanitaires et autochtones. », Anthropologie et Sociétés, vol. $27, \mathrm{n}^{\circ} 2$ (automne).

LAZAROU, J., B.H. POMERANZ et P.N. COREY (1998). «Incidence of adverse drug reactions in hospitalized patients. A metaanalysis of prospective studies », Journal of the American Medical Association, vol. 279 (avril), p. 1200-1205.

LUNA, L. E. (1986). Vegetalismo, Shamanism Among the Mestizo population of the Peruvian Amazon. Stockholm, Almqvist and Wiksell International.

PÉCOUL, B., P. CHIRAC, P. TROUILLER et J. PINEL (1999). "Access to essential drugs in poor countries. A lost battle?», JAMA, vol. 281, n 4, p. 361-367.

SANTOS, R. V. et E. A. COIMBRA Jr. (1994). Saùde e Povos Indigenas. Rio de Janeiro, Editora Fiocruz.

SCHULTES, R. et RAFFAUF (1990). The Healing Forest. Medicinal and Toxic Plants of the Northwest Amazonia, Portland, Oregon, Dioscorides Press.

VAN DER GEEST, S. et S. R. WHYTE (1989). "The charm of medicines: Metaphors and metonyms", Medical Anthropology Quarterly, International Journal for the Cultural and Social Analysis of Health, New Series, vol. 3, n 4, p. 345-367.

VIVEIROS DE CASTRO, E. (1992). From the Enemy's Point of View. Humanity and Divinity in an Amazonian Society. The University of Chicago Press.

WHYTE, S. R., S. VAN DER GEEST et A. HARDON (2002). Social Lives of Medicines. Cambridge University Press.

\section{Notes}

1. Le cours vise à former des dispensateurs locaux de biomédecine.

2. Les médecins, infirmières, infirmières auxiliaires des divers ONG ou du ministère de la Santé et de la Justice brésiliens et ceux qu'ils forment, les AIS.

3. Les chamans, homme-tabac, sages-femmes, herboristes issus de la tradition autochtone locale.

4. «Planta sabida» en portugais; désigne les plantes considérées comme dépositaires des savoirs et de la sagesse de ce monde (par opposition aux autres plantes médicinales qui tirent leur pouvoir de leur seule capacité de soulager le mal).

5. Cette recherche s'est effectuée dans le cadre d'un doctorat en anthropologie à l'Université Laval (Laplante, 2002), dont trois mois à titre de chercheure-volontaire MSF-H (décembre 1998-mars 1999).

6. <http://www.accessmed-msf.org/msf/ accessmed/accessmed.msf/html $>$, consulté en janvier 2001.

7. Dépliant de l'exposition-photos Too Poor to be Treated de la Campagne d'accès aux médicaments essentiels de MSF, février 2003.

8. La propriété intellectuelle autochtone est reconnue sous diverses formes par des accords internationaux (dont ceux de l'Organisation des Nations unies et de l'Organisation mondiale de la propriété intellectuelle); elle inclut le savoir, les pratiques, les convictions et la philosophie propres à chaque culture. Dans la pratique, les droits relatifs à la propriété intellectuelle sont menacés par d'autres accords internationaux, signés par les mêmes pays, mais qui protègent d'autres intérêts, tels ceux des accords de commerce, mais aussi ceux sous-jacents aux droits de l'homme qui promulguent un savoir technobiomédical universel. Il en résulte souvent une biopiraterie, à savoir une forme d'exploitation des ressources des communautés locales du Sud par des acteurs économiques divers qui, par le biais de biotechnologies et du système des brevets internationaux, protègent "leur découverte » et s'approprient le droit de regard sur la manière dont sera utilisée une plante ou une partie de plante. Cette exploitation touche non seulement aux ressources biologiques utilisées de manière traditionnelle dans les pays du Sud, mais aussi aux ressources intellectuelles de ces pays, c'est-à-dire à leurs savoirs et connaissances sur le monde vivant (Chomsky, 1994).

9. Dans ce modèle, la santé est recouvrée grâce à la biotechnologie elle-même, grâce à un produit miracle, un objet, une commodité, un remède tout-puissant qui est à lui seul responsable du «don» de la santé (Nichter, 1989).

10. Le signe de la présence des $\mathrm{M}-\mathrm{K}$ en cette région remonte à des documents ethnohistoriques du $\mathrm{XIX}^{\mathrm{e}}$ siècle. L'histoire est décousue à partir des épisodes violents de l'époque du caoutchouc (1821-1950) qui a suscité l'effritement et la dispersion des groupes autochtones. Le front extractiviste mercantile de seringueiros (extracteurs de caoutchouc) est signalé dans la région du Juruá en 1897. Les M-K qui habitent actuellement le territoire du lac Ualá seraient venus du haut Juruá du district Eirupepé en 1981 (Faulhaber, 1994).

11.Les enquêtes des anthropologues de la FUNAI et de la Petrobràs mettent en valeur les traditions liées aux ressources locales; les premières devant faciliter le processus d'homologation du territoire afin qu'il soit officiellement reconnu comme propriété autochtone, les secondes devant proposer des moyens de rétablir l'équilibre écologique rompu lors des projets d'extraction d'huile.

12. Selon les observations de Pollock (dans Santos et Coimbra, 1994), chez le peuple Kulina de l'État de l'Acre, en l'absence de conflits sociaux, il n'y a pas de cas de dori.

13. Viveiros de Castro (1992, p. 218) explique que chez les Arawete de l'Amazonie, les dieux et les morts sont les «choses musicales », ou les musiciens. Ils se manifestent par la chanson et leur véhicule est le chaman, qui est le «support des dieux». En référence à un chaman Jivaro de la Haute-Amazonie, Descola mentionne que «La musique de son tsayantar lui a permis de réveiller les fléchettes qu'il a stockées dans son corps...» (1993, p. 347).

14. Le rami est probablement le Banistériopsis caapi aussi appelé yage (Burroughs, 1989), ayahuasca (Schultes et Raffauf, 1990) ou visionary vine (Dobkin de Rios, 1972), l'écorce pour voir ou casca do pajé (l'écorce du chaman) chez les Kanamari. Son usage est noté pour traiter le parkinsonisme postencéphalique et des propriétés antimicrobiennes et antihelmintiques lui sont attribuées (Luna, 1986, p. 59). Selon des recherches pharmacalogiques, les alcaloïdes principaux de l'espèce Banisteriopsis, soit l'harmine, l'harmaline et le tetrahydroharmine, agissent sur le système nerveux central et sur le système nerveux autonomique et la plante serait un puissant dépuratif.

15. En Thaïlande, la résistance à la méfloquine serait apparue six ans seulement après qu'on l'eut introduite dans le pays (Pécoul et al., 1999, p. 364).

16. Ce serait aujourd'hui au tour d'un médicament chinois (tiré de la plante Artemisia апnua) de devenir le médicament vedette d'un protocole universel ou humanitaire pour lutter contre la malaria (Déclaration de l'OMS, endossée par MSF, Journée Afrique Malaria, 25 avril 2002).

17. La quinine, par exemple, est un alcaloïde de plante toxique que l'on trouve dans l'écorce de l'arbre Cinchona, originaire des Andes péruviennes et équatoriennes (voir le site du Centre de chimie IT de l'Université d'Oxford <http ://www.chem.ox.ac.uk/mom/ quinine/quinine.htm>, consulté en juin 2003).

18. Le fait que 100000 personnes meurent par année de médicaments "correctement prescrits» en Amérique du Nord (Lazarou et al., 1998) et combien d'autres de dépendances chroniques à ces produits ne semble pas ébranler l'utopie humanitaire. 